\title{
Topical antispasmodics during colonoscopy: do they have a role?
}

\section{(ㄷ)(우)}

\author{
Authors \\ Leonardo Zorron Cheng Tao Pu ${ }^{1,2}$, Rajvinder Singh ${ }^{1,2}$ \\ Institutions \\ 1 The University of Adelaide - School of Medicine \\ 2 Lyell McEwin Hospital - Gastroenterology, Adelaide, \\ South Australia
}

submitted 9.3.2017

accepted after revision 20.3.2017

\author{
Bibliography \\ DOI https://doi.org/10.1055/s-0043-106580 | \\ Endoscopy International Open 2017; 05: E408-E409 \\ (C) Georg Thieme Verlag KG Stuttgart · New York \\ ISSN 2364-3722
}

Corresponding author

Leonardo Zorron Cheng Tao Pu, The University of Adelaide

- School of Medicine, North Terrace Campus, Adelaide,

South Australia 5005, Australia

leonardo.zorronchengtaopu@adelaide.edu.au

leo.zorron@gmail.com
Colorectal cancer (CRC) is 1 of the 3 major causes of death by cancer worldwide. That is unfortunately a rather high (and unacceptable) incidence for a cancer with a known pathophysiology and effective screening methods. There are many factors responsible for this, including absence of any screening programs, poor uptake and compliance of patients within these programs, "missed" lesions in patients undergoing colonoscopy, and even the possibility of rapidly growing cancers. Other than ensuring that bowel preparation is immaculate, identification followed by systematic and complete removal of premalignant and early malignant colorectal lesions is paramount. In this edition of Endoscopy International Open, Togashi et al. evaluated the use of topical agents in order to aid endoscopic resection (ER) of premalignant colorectal lesions. It is known that even without endoscopic intervention, colonic spasms can have a negative impact on our performance during colonoscopy. That impact is much worse during complex ER techniques such as as endoscopic submucosal dissection (ESD) and endoscopic mucosal resection (EMR).

Some approaches have been tested to diminish colonic spasms during colonoscopies. Use of intravenous drugs to achieve a spasmolytic effect has been reported mostly with use of scopolamine and glucagon showing marginal to no benefit with regard to detection of colorectal polyps and cecal intubation [1-3]. Topical use of fluids such as warm water infusion and alverine citrate has been tested with debatable results. Although positive findings have been found including diminished pain [1] and quicker cecal intubation [2], the translation of these into clinical practice such as improved detection rate for colorectal polyps has not been demonstrated. Topical menthol was tested for colonic spasms as long ago as 35 years [3] and it has been adopted in some centers but mainly in the East $[4,5]$. Peppermint has also been used for upper endoscopy. Data from Japanese studies reported its efficacy and safety in mitigating gastric contractions [6-9]. Topical lidocaine has been used as a topical agent in several specialities manly due to its analgesic properties. However, use of topical lidocaine has not been described in colonoscopy for colonic spasms.

In this edition, Togashi et al. report the use of topical lidocaine in patients randomized to it or topical peppermint for durability of inhibiting colonic spasms and for rebound spasms. The authors report a positive effect in both arms (29 of 30 patients) but with a more lasting effect with the former. Unfortunately, this study only observed the effect of a single dose of the solution $(30 \mathrm{~mL})$ for 5 minutes. That provides us with valuable but perhaps limited information with regard to the applicability of topical lidocaine in routine practice. Evaluating repetitive doses during an entire ER procedure would have provided us with a more direct answer about its use. In addition, evaluation of clinically applicable measures such as differences in complications and detection rate of polyps would have been a valuable addition. Nevertheless these results are very promising. A more "calm" bowel during diagnostic and therapeutic colonoscopy is desirable. Future research could concentrate on the use of topical lidocaine and its impact on adenoma and sessile serrated adenoma/polyp detection rates, and the potential positive effect on completeness of resection. Togashi et al. have indeed provided a very fertile field which should be explored further in coming years. 


\section{Competing interests}

None

\section{References}

[1] Rabenstein T, Radaelli F, Zolk O. Warm water infusion colonoscopy: a review and meta-analysis. Endoscopy 2012; 44: 940 - 951

[2] Altintas E, Ucbilek E, Sezgin O et al. Alverine citrate plus simethicone reduces cecal intubation time in colonoscopy - a randomized study. Turk J Gastroenterol 2008; 19: 174-179

[3] Leicester R, Hunt R. Peppermint oil to reduce colonic spasm during endoscopy. The Lancet 1982; 320: 989

[4] Asao T, Mochiki E, Suzuki H et al. An easy method for the intraluminal administration of peppermint oil before colonoscopy and its effectiveness in reducing colonic spasm. Gastrointest Endosc 2001; 53: $172-177$
[5] Yoshida N, Naito Y, Hirose R et al. Prevention of colonic spasm using L-menthol in colonoscopic examination. Int J Colorectal Dis 2014; 29: $579-583$

[6] Hiki N, Kaminishi M, Hasunuma T et al. A phase I study evaluating tolerability, pharmacokinetics, and preliminary efficacy of L-menthol in upper gastrointestinal endoscopy. Clin Pharmacol Therap 2011; 90: $221-228$

[7] Hiki N, Kaminishi M, Yasuda K et al. Multicenter phase II randomized study evaluating dose-response of antiperistaltic effect of L-menthol sprayed onto the gastric mucosa for upper gastrointestinal endoscopy. Digest Endosc 2012; 24: 79-86

[8] Yamamoto N, Nakai Y, Sasahira $\mathrm{N}$ et al. Efficacy of peppermint oil as an antispasmodic during endoscopic retrograde cholangiopancreatography. J Gastroenterol Hepatol 2006; 21: 1394 - 1398

[9] Fujishiro M, Kaminishi M, Hiki N et al. Efficacy of spraying I-menthol solution during endoscopic treatment of early gastric cancer: a phase III, multicenter, randomized, double-blind, placebo-controlled study. J Gastroenterol 2014; 49: 446-454 